\title{
Indice de masa corporal y percepción de la imagen corporal en una población adulta mexicana: la precisión del autorreporte
}

Ignacio 0 suna-Ramírez, M en $C_{,}{ }^{(1)}$ Bernardo Hernández-Prado, PhD, ${ }^{(2)}$ Julio César Campuzano, Dr en $C$, (2) Jorge Salmerón, PhD. ${ }^{(3)}$

\section{Osuna-Ramírez I, Hernández-Prado B, Campuzano JC, Salmerón J. Indice de masa corporal y percepción de la imagen corporal en una población adulta mexicana: la precisión del autorreporte. Salud Publica Mex 2006;48:94-103.}

\section{Resumen}

Objetivo. Evaluar la precisión del autorreporte de índice de masa corporal y de la percepción de la imagen corporal en población adulta mexicana. Material y métodos En 1998, en el estado de Morelos, M éxico, trabajadores del Instituto Mexicano del Seguro Social participaron en el "Estudio de cohorte del IMSS", de tipo longitudinal, y respondieron un cuestionario auto aplicado, diseñado para recabar una gran variedad de factores de riesgo para enfermedades crónicas con base en estilos de vida. Los participantes auto rreportaron su imagen corporal seleccionando la silueta que mejor les representaba, entre un juego de siluetas numeradas del 1 al 9. Asimismo, informaron su peso y talla actuales, sin saber que posteriormente habría un seguimiento de medidas directas de peso y talla. Entre 4 y 8 meses después se pesó y midió a los participantes bajo procedimientos estandarizados. Se calcularon correlaciones Spearman para analizar la correlación entre los datos autorreportados y las medidas to madas. Se utilizó la prueba Kruskal-W allis para evaluar la magnitud de la diferencia entre la talla, el peso y el IMC reportados y los medidos (medido menos autorreportado), por nivel de educación y por rango de edad. Se utilizó regresión robusta para evaluar el efecto potencial de las características específicas individuales de la diferencia del peso y de la talla, tanto en los medidos como en los

\author{
Osuna-Ramírez I, Hernández-Prado B, \\ Campuzano JC, Salmerón J. \\ Body mass index and body image \\ perception in Mexican adult population: \\ The accuracy of self-reporting. \\ Salud Publica Mex 2006;48:94-103.
}

\begin{abstract}
A bstract
Objective To evaluate the accuracy of the self-reported body mass index and body image perception in a population of Mexican adults. Material and Methods In 1998, in the state of Morelos, Mexico, participants in the longitudinal study of Mexican Social Security Institute workers "IMSS Cohort Study" responded to a baseline, self-administered questionnaire designed to collect a large variety of lifestyle risk factors for chronic diseases. Participants self-reported their body image by selecting the silhouette that best portrayed them, from a set of silhouettes ranked from 1 to 9 . Participants also self-reported their current weight and height, unaware that direct measurements of weight and height were to follow. Four to eight months later participants were weighed and their heights were measured using standardized procedures. Spearman correlations were computed to analyze the correlation between self-reported and measured data. The Kruskal-W allis test was used to evaluate the magnitude of difference between measured and selfreported height, weight and body mass index (BMI) (measured minus self-reported), by educational level and catego ries of age. Robust regression was used to evaluate the potential effect of specific individual characteristics on differences between measured and self-reported weight and height. Logistic regression analysis was used to evaluate the
\end{abstract}

Este estudio fue apoyado con la beca número 125975/129211 del Consejo N acional de Ciencia y Tecnología (CO N ACyT) y por el Instituto Mexicano del Seguro Social, México.

(1) Facultad de Ciencias Q uímico Biológicas, Universidad Autónoma de Sinaloa, Culiacán, Sinaloa, México.

(2) Instituto $\mathrm{N}$ acional de Salud Pública, Cuernavaca, Morelos, México.

(3) Unidad de Investigación Epidemiológica y en Servicios de Salud, Instituto Mexicano del Seguro Social, Morelos, México.

Fecha de recibido: 8 de marzo de 2005 - Fecha de aprobado: 28 de octubre de 2005

Solicitud de sobretiros: M en C. Ignacio 0 suna Ramírez. Calle Solón Sabre Morrel 3115, Fraccionamiento Universidad 94 etapa 2,80090 Culiacán, Sinaloa, México.

Correo electrónico: nachoor@ uas.uasnet.mx 
reportados. Se hizo análisis de regresión logística para evaluar la sensibilidad, especificidad y valores predictivos para el índice de masa corporal. Resultados El estudio incluyó a 934 individuos, de los cuales $62.6 \%$ fueron mujeres; éstas tuvieron una media de talla de $1.55 \mathrm{~m} \pm 0.06$ y un peso de $65.4 \mathrm{~kg} \pm 10.9$, mientras que en los hombres la media de talla fue de $1.67 \mathrm{~m} \pm 0.06$ y el peso de $77.7 \mathrm{~kg} \pm 12.5$. La media del IMC fue de $27.4 \mathrm{~kg} / \mathrm{m}^{2} \pm 4.3$ (mujeres: $27.2 \mathrm{~kg} / \mathrm{m}^{2} \pm 4.45$; hombres: $27.8 \pm 3.87$ ). La mediana de la percepción de la imagen corporal fue 5 (percentil $25=4$; percentil $75=6$ ). Las correlaciones entre la talla, el peso y el IMC medidos y auto rrepo rtados para to dos los sujetos fueron de $0.94,0.96$, y 0.90, respectivamente. La correlación entre el IMC y la percepción de la imagen corporal fue de 0.64 (0.67 para las mujeres, y 0.59 para los hombres). La media de lo autorreportado no tuvo variación mayor de $1.3 \mathrm{~cm}$ con la talla medida, y no más de $3.17 \mathrm{~kg}$ con el peso medido. Los errores de estimaciones de peso, talla e IMC disminuyeron en relación con el nivel de educación. La sensibilidad y la especificidad, antes de ajustar el autorreporte del IMC con las categorías de sobrepeso y obesidad que se colapsaron en una, fueron 94.8 y $83.0 \%$, respectivamente. Para la percepción de la imagen corporal esos valores fueron de 87.6 y $48.9 \%$. Discusión. Los resultados de este trabajo sugieren que el IMC y la percepción de la imagen corporal autorreportados pueden ser indicado res útiles acerca de la condición de sobrepeso en adultos mexicanos. Las sensibilidades y especificidades asociadas con las categorías autorreportadas corregidas de IMC pueden utilizarse para ajustar razones de momios y riesgos relativos, calculados de los niveles derivados del IMC autorreportado. La percepción de imagen corporal permitió la correcta clasificación de individuos con IMC $\geq 25$, y mostró que puede ser un estimado válido para utilizarse en estudios epidemiológicos.

Palabras clave: índice de masa corporal; imagen corporal; autorreporte; México sensitivity, specificity, and predictive values for BMI. Results The study included 934 subjects, of which $62.6 \%$ were female. Females had a mean measured height of $1.55 \mathrm{~m}$ (SD 0.06 ) and weight of $65.4 \mathrm{~kg}$ (SD 10.9), while males had a mean height of $1.67 \mathrm{~m}$ (SD 0.06) and weight of $77.7 \mathrm{~kg}$ (SD 12.5). The mean BMI was $27.4 \mathrm{~kg} / \mathrm{m}^{2}$ (SD 4.3) (females: 27.2 $\mathrm{kg} / \mathrm{m}^{2} \pm 4.45$; males: $\left.27.8 \pm 3.87\right)$. The median of body image perception (BIP) was $5\left(25^{\text {th }}\right.$ percentile $=4 ; 75^{\text {th }}$ percentile $\left.=6\right)$. Correlations between measured and self-reported height, weight, and BMI for all subjects were 0.94, 0.96, and 0.90, respectively. The correlation between BMI and BIP was 0.64 ( 0.67 for females and 0.59 for males). Self-reported mean varied no more than $1.3 \mathrm{~cm}$ from measured height and no more than $3.17 \mathrm{~kg}$ from measured weight. Error estimations of height, weight, and BMI decreased with educational level. Sensitivity and specificity before adjusting self-reported BMI with overweight and obesity categories that were collapsed into one were 94.8 and $83.0 \%$, respectively; for BIP, those values were 87.6 and $48.9 \%$. Conclusions The results suggest that self-reported BMI and BIP can be useful indicators of an overweight condition in Mexican adults. The sensitivities and specificities associated with corrected selfreported BMI categories could be used to adjust odds ratios and relative risks, calculated from BMI levels derived from the self-reported BMI. Body image perception made it possible to correctly classify individuals with $B M I \geq 25$, showing that it may be a valid estimate for use in epidemiological surveys.

Key words: body mass index; body image; self-reported; Mexico
L a obesidad es uno de los principales determinantes de la salud en adultos y un fenómeno mundial que incluye tanto a los países industrializados como a los países en desarrollo. ${ }^{1}$

Debido al gran impacto que tiene la obesidad en la mayoría de las enfermedades crónicas, ${ }^{2-5}$ es necesario evaluarla en cada estudio sobre los determinantes de la salud en poblaciones de adultos. Con frecuencia, los estudios epidemiológicos sobre enfermedades crónicas recopilan datos a través de cuestionarios autoaplicados.

No siempre es factible tomar las medidas directas de talla y peso en estudios a gran escala, ya que requieren equipo costoso y personal capacitado; por tanto, se han sustituido con las medidas de peso y talla autorreportadas desde los años 70. ${ }^{6,7}$

Otra alternativa es autorreportar la imagen corporal usando siluetas estándar que representan la percepción corporal en diferentes etapas de la vida. ${ }^{7,8}$ Asimismo, se ha sugerido que el autorreporte de la percepción de la imagen corporal (PIC) puede ser un buen sustituto como indicador del índice de masa corporal (IMC). Sin embargo, la percepción de la imagen varía entre las diferentes poblaciones. ${ }^{4}$

Cuando se utiliza información autorreportada en estudios epidemiológicos, es necesario asegurar su validez y confiabilidad. ${ }^{6} \mathrm{Al}$ utilizar datos autorreportados como una base para un seguimiento subsiguiente, se debe tomar en cuenta la magnitud de una posible diferencia y de un error aleatorio. . $^{6,9,10}$

Algunos investigadores han mostrado la precisión de peso y talla autorreportados en poblaciones específicas. ${ }^{6,11-15}$ Sin embargo, en México es escasa la experiencia en la utilización de cuestionarios autoaplicados para obtener datos de peso y talla. ${ }^{15-21}$ Por tanto, es necesario validar este método en la población mexicana. 
En este estudio se evaluó la precisión del IMC calculado a partir del peso y de la talla autorreportados, referido en todo el texto como IMC autorreportado, con respecto al IMC obtenido de los valores medidos y la precisión de la PIC relativa a las categorías en que se clasifica comúnmente el IMC para denotar peso normal, sobrepeso y obesidad.

\section{Material y métodos}

Se utilizaron datos obtenidos en el "Estudio de cohorte del Instituto Mexicano del Seguro Social -IMSS-" iniciado en 1998 en el estado de Morelos, México. La población base, distribuida en cuatro zonas -Hospital General Regional 1; Cuautla y sus municipios; Unidad Médica Familiar 20, Delegación y sus municipios y Zacatepec-, fue de 5706 trabajadores del IMSS entre 18 y 89 años de edad. De la población estudiada, 39.8\% correspondió al sexo femenino. Con la finalidad de incrementar el índice de participación, se llevaron a cabo pláticas para los individuos participantes, exponiendo los objetivos del estudio y haciendo hincapié en los beneficios que podría aportar la investigación. A cada trabajador se le entregó un sobre sellado que contenía una carta de invitación personal para partici- par en el estudio, así como la forma de mantener la confidencialidad de la información proporcionada y un cuestionario autoaplicable.

Entre el 1 de noviembre de 1998 y el 30 de abril de 1999, un total de 2907 participantes conformaron la cohorte del IMSS; respondieron a un cuestionario autoaplicado diseñado para obtener una amplia variedad de factores de riesgo para enfermedades crónicas, tomando como base estilos de vida.

Este cuestionario incluyó una detallada estimación de la imagen corporal mediante la selección de la silueta que mejor representaba la imagen del participante, en un juego de nueve siluetas, en rangos que iban desde muy delgada hasta extremadamente obesa (figura 1). En 1970, Sorensen y colaboradores ${ }^{7}$ propusieron siluetas a través de las cuales los participantes identificaban a sus padres; debido a que dichas siluetas correspondían a una población distinta a la mexicana, se procedió a evaluar su idoneidad en la autopercepción de la imagen corporal, con el fin de adoptarlas para población mexicana. El cuestionario también incluyó autorreportar el peso y la talla actuales. Los participantes desconocían que, entre cuatro y ocho meses después de la autoaplicación del cuestionario, se tomarían las medidas directas de peso y talla. Debido a la logística

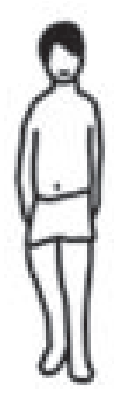

1

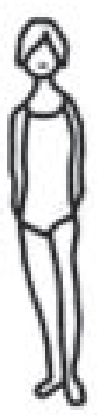

1

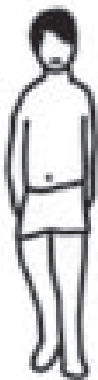

2

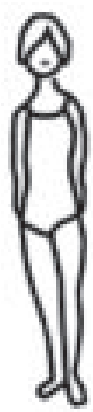

2

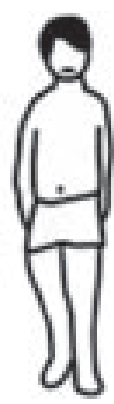

3

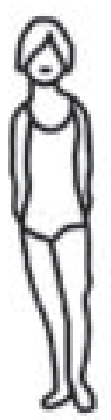

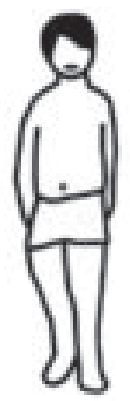

4

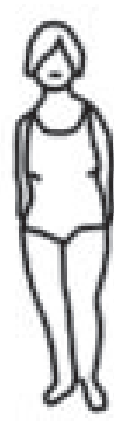

4

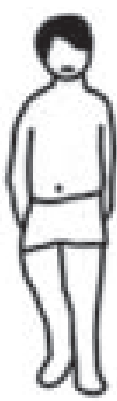

5

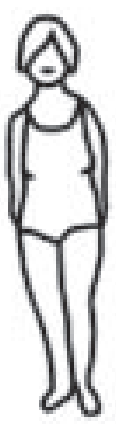

5
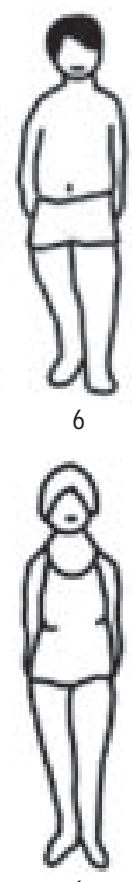

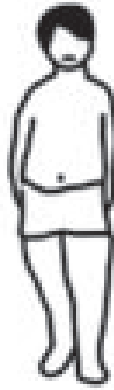

7

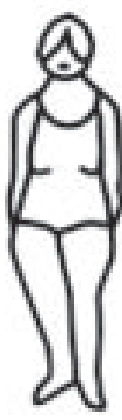

7

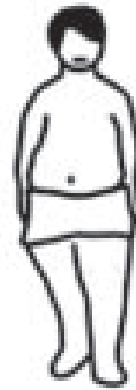

8

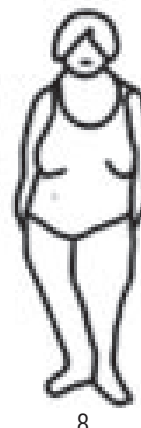

8

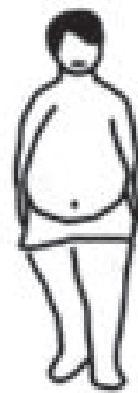

9

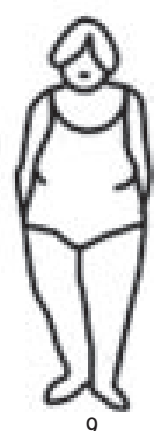

Figura 1. Dibujos de siluetas de las QUe los participantes eligieron la QUe mejor les representa 
del estudio fue imposible hacer esas mediciones al momento del autorreporte de peso y talla. No obstante, dada la naturaleza de los participantes -adultos- este tiempo no afectaría en gran medida los resultados para la talla, y para controlar la probable variación del peso se incluyó una pregunta referente a si había cambiado de peso en el último año y que fue considerada en el análisis. Antropometristas capacitados y estandarizados se encargaron de tomar las medidas. La talla se tomó al $0.5 \mathrm{~cm}$ más cercano sin zapatos, usando una vara métrica fija sobre el muro. Se pesó a los individuos con ropa ligera (sin saco, suéter ni zapatos), tomando el $0.1 \mathrm{~kg}$ más cercano y usando una báscula estándar, calibrada con pesos estándar al inicio de cada sesión de toma de medidas. Una vez registradas estas medidas, se procedió a calcular el IMC 1 [peso $(\mathrm{kg}) /$ talla $\left.^{2}\left(\mathrm{~m}^{2}\right)\right]^{22}$ de ambas medidas: las tomadas directamente y las autorreportadas para peso y talla. Para evaluar la sensibilidad, la especificidad y los valores predictivos del IMC y de la PIC reportados versus índice de imagen corporal medido, se analizaron diferentes categorías del IMC, y se adoptó la establecida por la Organización Mundial de la Salud $(\mathrm{OMS})^{23-25}\left(<25,25-30\right.$ y $\left.\geq 30 \mathrm{~kg} / \mathrm{m}^{2}\right)$, así como de percepción de imagen corporal (dibujos 1-3, 4-6 y 7-9 de la figura 1, para normal, sobrepeso y obesidad, respectivamente). Las categorías de índice de percepción de imagen corporal para mujeres y hombres se validaron mediante el cálculo de sensibilidad y especificidad, tomando como contraste las categorías del IMC establecidas por la OMS.

Para los propósitos del presente estudio, se analizaron datos de una submuestra de 934 hombres y mujeres no embarazadas, todos participantes del "Estudio de cohorte del IMSS". Dicha submuestra quedó conformada con aquellos participantes que entregaron el cuestionario contestado y a quienes, además, se les tomaron las mediciones de peso y talla. Las edades se tomaron en años y las categorías de educación fueron primaria, secundaria, preparatoria, estudios profesionales y de posgrado. El estado civil se clasificó como casados/unión libre, viudos, separados/divorciados y nunca casados. Las categorías de ocupación fueron pensionados; asistentes de enfermería y de médicos; médicos; administrativos; empleados de intendencia; trabajadores sociales y entrenadores físicos; estudiantes y escolares; nutriólogos, químicos y farmacéuticos y personal de apoyo. La modificación en el peso durante el año anterior se obtuvo haciendo la pregunta: “¿cambió su peso en el año anterior?"

\section{A nálisis estadístico}

Se estimaron los promedios y las desviaciones estándar, así como la distribución de las diferencias entre los datos (peso, talla, IMC y PIC) medidos y los autorreportados. Se hicieron gráficas para desplegar las distribuciones del IMC por variables categóricas. Se computaron correlaciones de Spearman para analizar la correlación entre el peso, la talla y el IMC autorreportados y medidos, y entre el IMC y la PIC. ${ }^{26}$ Asimismo, se obtuvieron correlaciones entre el peso y la talla medidos y autorreportados por niveles del IMC (normal, sobrepeso y obesidad).

Se utilizó la prueba Kruskal-Wallis ${ }^{26}$ para evaluar la magnitud de la diferencia entre las medidas de peso y talla tomadas clínicamente y las autorreportadas por nivel de educación y por categoría de edad, así como las diferencias en el IMC (las medidas menos las autorreportadas). ${ }^{27}$ La regresión robusta ${ }^{28}$ se realizó en una submuestra de 506 participantes elegidos aleatoriamente que representaron $54 \%$ del total de la población del estudio, con el fin de evaluar el efecto potencial de características específicas de los individuos sobre la diferencia del peso, la talla y el IMC autorreportados, comparados con las medidas directas en esta submuestra. El promedio de las diferencias de peso, talla e IMC (medidos menos reportados) se comparó con el análisis de varianza no paramétrica, ${ }^{26}$ usando como covariables sexo, grupo de edad, nivel de educación, estado civil y ocupación. Para validar tanto las categorías del IMC autorreportado como la percepción de la imagen corporal, se utilizó el análisis de regresión logística, ${ }^{29-32}$ a fin de evaluar la sensibilidad y la especificidad, tomando como contraste el IMC medido clínicamente y utilizando las categorías establecidas por la OMS.

Con el fin de corregir el autorreporte de peso y talla, se propuso un modelo de regresión lineal tomando como variable de respuesta las diferencias de las mediciones clínicas y el autorreporte de peso y talla, en función de las covariables que explicaban tales diferencias. Una vez estimados los coeficientes de las covariables que resultaron estadísticamente significativas, se aplicó el modelo al resto de la muestra para evaluar la mejoría en los estimados autorreportados. La correlación y los valores de sensibilidad-especificidad se calcularon para corregir los datos autorreportados y los medidos de talla, peso e IMC.

A menos que se mencione algo diferente, el nivel de significancia utilizado en este artículo fue de $p<0.05$. Todos los análisis estadísticos se realizaron utilizando el programa Stata versión 6.0.

\section{Resultados}

Se analizaron los datos de 934 participantes con edades entre 18 y 76 años (media 42.3 años; $D E$ 8.5). Las mujeres conformaron $62.6 \%$ de la muestra $(n=585)$; 
la media de talla y peso medidos fue de $1.55 \mathrm{~m} \pm 0.06 \mathrm{y}$ $65.4 \mathrm{~kg} \pm 10.9$, respectivamente. Los hombres tuvieron una media de talla medida de $1.67 \mathrm{~m} \pm 0.06$ y peso de $77.7 \mathrm{~kg} \pm 12.5$. La media del IMC medido fue de 27.4 $\mathrm{kg} / \mathrm{m}^{2} \pm 4.33$ (mujeres: $27.2 \mathrm{~kg} / \mathrm{m}^{2} \pm 4.45$; hombres: 27.8 \pm 3.87 ) (cuadro I). La mediana de la PIC autorreportada a través de la selección de siluetas fue de 5 (percentil $25=4$ y percentil $75=6$ ). La media del IMC medido mostró un incremento de la tendencia a través de los niveles de la PIC (datos no mostrados). La prevalencia de obesidad fue de $23.5 \%$ (mujeres $21.5 \%$ y hombres $26.6 \%$ ) para el IMC $\geq 30$, y $23.9 \%$ para la PIC $\geq 7$ (mujeres $21.2 \%$ y hombres $28.4 \%$ ). De manera simi- lar, la prevalencia del IMC $\geq 25$ fue de $70 \%$ (mujeres $66.8 \%$ y hombres $75.3 \%$ ) y para la PIC $\geq 4$ fue de $76.6 \%$ (mujeres $74.9 \%$ y hombres $79.6 \%$ ).

\section{Talla autorreportada}

La correlación global entre la talla medida y la autorreportada fue de 0.94 . Las correlaciones en las diferentes categorías del IMC medido $(<25,25-30$ y $\geq 30$ $\mathrm{kg} / \mathrm{m}^{2}$ ) no tuvieron cambios sensibles; éstas fueron de 0.87 para las mujeres y de 0.90 para los hombres (cuadro II). La media de las diferencias entre talla medida y autorreportada fue de -0.013 ( $p<0.001) ; 62.4 \%$ de los

\section{Cuadro I}

\section{Características sociodemográficas de los participantes del Estudio de Cohorte del Instituto Mexicano del Seguro Social, Morelos, México, 1998}

\begin{tabular}{|c|c|c|c|c|c|c|}
\hline Características & $\%$ & $\begin{array}{c}\text { Media } \\
\text { Edad (años) }\end{array}$ & Talla (m) & $\frac{\text { edia medi }}{\text { Peso }(\mathrm{kg})}$ & IMC $\left(\mathrm{kg} / \mathrm{m}^{2}\right)$ & $\begin{array}{c}\text { Mediana } \\
\text { PIC selección de silueta }\end{array}$ \\
\hline \multicolumn{7}{|l|}{ Sexo } \\
\hline Mujeres & 62.6 & 42.0 & 1.55 & 65.4 & 27.2 & 5.0 \\
\hline Hombres & 37.4 & 42.9 & 1.67 & 77.7 & 27.8 & 5.0 \\
\hline \multicolumn{7}{|l|}{ Edad } \\
\hline 18-39 & 35.2 & 33.5 & 1.60 & 67.5 & 26.3 & 4.0 \\
\hline $40-59$ & 62.3 & 46.4 & 1.60 & 71.5 & 28.1 & 5.0 \\
\hline $60-76$ & 2.5 & 65.0 & 1.55 & 68.2 & 28.2 & 6.0 \\
\hline
\end{tabular}

\begin{tabular}{lrrrrr}
$\begin{array}{l}\text { N ivel de educación } \\
\text { Primaria }\end{array}$ & 8.5 & 51.0 & 1.60 & 74.0 & 6.0 \\
\hline Secundaria & 22.1 & 44.9 & 1.58 & 71.2 & 28.7 \\
\hline Preparatoria & 25.1 & 38.9 & 1.60 & 68.3 & 26.6 \\
\hline Profesional/posgrado & 44.3 & 41.3 & 1.60 & 69.6 & 21.2
\end{tabular}

\begin{tabular}{lrrrrrr}
$\begin{array}{l}\text { Estado civil } \\
\text { Casados/unión libre }\end{array}$ & 74.5 & 42.1 & 1.60 & 71.6 & 27.8 & 5.0 \\
\hline Viudos & 2.3 & 54.0 & 1.53 & 64.6 & 27.6 & 26.8 \\
\hline Separados/divorciados & 9.3 & 43.9 & 1.57 & 66.0 & 4.0 \\
\hline N unca casados & 13.9 & 40.3 & 1.58 & 65.3 & 26.2
\end{tabular}

\begin{tabular}{|c|c|c|c|c|c|c|}
\hline Pensionados & 3.1 & 60.5 & 1.54 & 67.0 & 28.0 & 6.5 \\
\hline Enfermeras & 15.1 & 42.6 & 1.54 & 67.0 & 28.4 & 5.0 \\
\hline Asistentes médicos & 10.9 & 42.6 & 1.56 & 65.2 & 26.9 & 5.0 \\
\hline Personal administrativo & 30.4 & 40.1 & 1.61 & 70.3 & 27.1 & 5.0 \\
\hline Empleados multitareas & 20.3 & 43.1 & 1.63 & 70.0 & 28.1 & 5.0 \\
\hline 0 tros & 20.2 & 41.6 & 1.61 & 74.8 & 26.9 & 5.0 \\
\hline
\end{tabular}

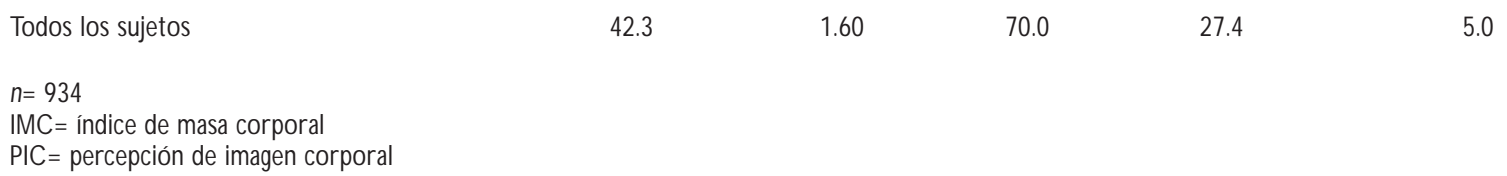




\section{Cuadro II Correlación entre datos Medidos Y AUTORREPORTADOS. ESTUDIO DE COHORTE del Instituto Mexicano del Seguro Social, Morelos, México, 1998}

\begin{tabular}{|c|c|c|c|c|c|}
\hline \multirow[b]{2}{*}{ Características } & \multicolumn{4}{|c|}{$n=934$} & \multirow{2}{*}{$\begin{array}{c}n=428 \\
\text { IMC corregido }\end{array}$} \\
\hline & Talla & Peso & IMC & PIC* & \\
\hline \multicolumn{6}{|l|}{ Sexo } \\
\hline Mujeres & 0.87 & 0.94 & 0.89 & 0.67 & 0.91 \\
\hline Hombres & 0.90 & 0.96 & 0.91 & 0.59 & 0.93 \\
\hline \multicolumn{6}{|c|}{ Indice de masa corporal } \\
\hline$<25$ & 0.94 & 0.92 & 0.72 & 0.40 & 0.74 \\
\hline $25-30$ & 0.94 & 0.93 & 0.68 & 0.29 & 0.66 \\
\hline$\geq 30$ & 0.94 & 0.95 & 0.75 & 0.36 & 0.73 \\
\hline \multicolumn{6}{|l|}{ Edad } \\
\hline 18-39 & 0.94 & 0.95 & 0.89 & 0.60 & 0.90 \\
\hline $40-59$ & 0.94 & 0.96 & 0.91 & 0.66 & 0.93 \\
\hline $60-76$ & 0.66 & 0.96 & 0.62 & 0.41 & 0.79 \\
\hline
\end{tabular}

Nivel de educación

\begin{tabular}{llllll} 
Primaria & 0.90 & 0.96 & 0.83 & 0.43 & 0.92 \\
\hline Secundaria & 0.93 & 0.96 & 0.89 & 0.65 & 0.93 \\
\hline Preparatoria & 0.94 & 0.95 & 0.87 & 0.61 & 0.84 \\
\hline Profesional/posgrado & 0.94 & 0.96 & 0.91 & 0.66 & 0.93
\end{tabular}

Estado civil

\begin{tabular}{lrrrrr} 
Casados/unión libre & 0.94 & 0.96 & 0.90 & 0.64 & 0.91 \\
\hline Viudos/separados/divorciados & 0.85 & 0.92 & 0.85 & 0.52 & 0.86 \\
\hline N unca casados & 0.95 & 0.97 & 0.92 & 0.74 & 0.95
\end{tabular}

O cupación

\begin{tabular}{llllll} 
Pensionados & 0.75 & 0.97 & 0.74 & 0.76 & 0.85 \\
\hline Enfermeras & 0.87 & 0.95 & 0.91 & 0.73 & 0.92 \\
\hline Asistentes médicos & 0.93 & 0.92 & 0.85 & 0.68 & 0.86 \\
\hline Administrativos & 0.93 & 0.96 & 0.92 & 0.66 & 0.93 \\
\hline Empleados multitareas & 0.94 & 0.97 & 0.90 & 0.61 & 0.91 \\
\hline Otros & 0.95 & 0.96 & 0.91 & 0.60 & 0.91 \\
Total & & & & & \\
& 0.94 & 0.96 & 0.90 & 0.64 & 0.91
\end{tabular}

* PIC versus IMC medido

IMC = índice de masa corporal

$\mathrm{PIC}=$ percepción de imagen corporal

participantes sobrestimaron su talla (media de la diferencia $0.028 \mathrm{~m} \pm 0.025$ ) y $21.7 \%$ la subestimaron (media de la diferencia $0.020 \mathrm{~m} \pm 0.022$ ). No hubo significancia estadística en la diferencia de estimación por sexo (mujeres -0.013 y hombres $-0.014 ; p=0.46$ ), ocupación $(p=0.19)$, nivel de educación $(p=0.18)$ y estado civil $(p=0.13)$.

Empero, se observaron diferencias entre la talla medida y la autorreportada entre grupos de edad $(p=0.02)$. La sobrestimación de talla aumentó con la edad (-0.010 m en el grupo de 18-29 años y $-0.045 \mathrm{~m}$ para el de 60-76 años; $p=0.003$ ). La magnitud de la diferencia en la talla disminuyó a través de las categorías del IMC medido (-0.006, -0.014 y -0.019 m para IMC $<25 ; 25-30$ y $\geq 30 \mathrm{~kg} / \mathrm{m}^{2}$, respectivamente).

\section{Peso autorreportado}

La correlación entre el peso medido y el autorreportado fue de 0.96 . Las correlaciones dentro de las diferentes categorías del IMC medido $(<25 ; 25-30$ y $\geq 30$ $\mathrm{kg} / \mathrm{m}^{2}$ ) fueron de $0.92,0.93$ y 0.95 , respectivamente; tales correlaciones fueron de 0.94 para mujeres y 0.96 para hombres (cuadro II). La media de la diferencia entre el peso medido y el autorreportado fue estadísticamente significativo (media de la diferencia $1.41 \mathrm{~kg}$; $p<0.001$ ). De los participantes, $24 \%$ sobrestimaron su peso (media $-2.36 \mathrm{~kg} \pm 2.07$ ) y $62.4 \%$ lo subestimaron (media $3.17 \mathrm{~kg} \pm 2.44$ ). Hubo una diferencia estadísticamente significativa en la precisión de la estimación entre el peso medido y el autorreportado por sexo ( $p=0.04$ ); la media fue de $1.26 \pm 3.15$ para las mujeres, y de $1.68 \mathrm{~kg} \pm 3.32$ para los hombres. Las estimaciones de las mujeres fueron mejores que las de los hombres en ambos casos, tanto para los que subestimaron su peso ( 2.98 versus $3.48 \mathrm{~kg}, p=0.004$ ) como para quienes lo sobrestimaron $(-2.25$ versus $-2.59 \mathrm{~kg}, p=0.09)$.

Las diferencias entre el peso medido y el autorreportado por ocupación y por estado civil fueron marginalmente significativas ( $p=0.07)$. La distribución de la diferencia en la estimación del peso mostró una tendencia positiva a lo largo de los diferentes niveles del IMC medido. La desviación estándar del error reportado aumentó con la categoría del IMC. La magnitud de la diferencia entre lo medido menos lo autorreportado, representando el error en el estimado autorreportado, se incrementó junto con el aumento del IMC medido $(0.15,1.39$ y $3.08 \mathrm{~kg}$ para IMC $<25 ; 25-30$ y $\geq 30$ $\mathrm{kg} / \mathrm{m}^{2}$, respectivamente).

\section{Indice de masa corporal autorreportado}

La correlación entre el IMC medido y el autorreportado fue de 0.90; dentro de los diferentes niveles del IMC medido $\left(<25,25-30\right.$ y $\left.\geq 30 \mathrm{~kg} / \mathrm{m}^{2}\right)$ fue de $0.72,0.68$ y 0.75 , respectivamente; tales correlaciones fueron de 0.89 para las mujeres y de 0.91 para los hombres (cuadro II). 
La media de la diferencia entre el IMC medido y el autorreportado fue estadísticamente significativa $(p<0.001)$. Un total de $24.5 \%$ de los participantes sobrestimaron el IMC (media $-0.93 \mathrm{~kg} / \mathrm{m}^{2} \pm 0.88$ ) y $72.6 \%$ lo subestimaron (media $1.67 \mathrm{~kg} / \mathrm{m}^{2} \pm 1.40$. No hubo diferencias estadísticamente significativas en la precisión de la estimación entre el IMC por sexo (media 0.95 $\mathrm{kg} / \mathrm{m}^{2} \pm 1.75$ para mujeres, y de $1.04 \mathrm{~kg} / \mathrm{m}^{2} \pm 1.61$ para hombres; $p=0.32$ ).

No hubo diferencia en la precisión de la estimación del IMC por sexo; para todas las edades combinadas, las estimaciones de los hombres fueron menos precisas que las de las mujeres (media de la diferencia 1.04 para los hombres, $0.95 \mathrm{~kg} / \mathrm{m}^{2}$ para las mujeres), tanto entre quienes subestimaron el IMC (1.69 versus $\left.1.65 \mathrm{~kg} / \mathrm{m}^{2}, p=0.92\right)$ como entre quienes lo sobrestimaron $\left(-0.97\right.$ versus $\left.-0.86 \mathrm{~kg} / \mathrm{m}^{2}, p=0.56\right)$.

La subestimación del IMC disminuyó con el nivel de educación ( $p=0.56$ ). La diferencia entre el medido y el autorreportado dentro de las categorías de ocupación fue estadísticamente significativa $(p=0.01)$, mientras que por estado civil fue sólo marginalmente significativo $(p=0.09)$. La subestimación fue menor en el personal administrativo $\left(0.8 \mathrm{~kg} / \mathrm{m}^{2}\right)$ y mayor en los pensionados retirados $\left(1.82 \mathrm{~kg} / \mathrm{m}^{2}\right)$.

La distribución de la diferencia en el IMC mostró una tendencia positiva a través de sus categorías medidas. La desviación estándar del error reportado se incrementó junto con el aumento del IMC; la diferencia en la magnitud de la desviación estándar (indicando mayor variabilidad en el error de estimación) se acompañó de un cambio en la ubicación y una distorsión en la dirección de la subestimación, indicando mayor diferencia. El grado de clasificación errónea entre el IMC medido y el autorreportado varió entre sus diferentes categorías (cuadro III). La prevalencia de obesidad fue de $23.5 \%$ (mujeres $21.5 \%$ y hombres $26.6 \%$ ) para el IMC medido $\geq 30 \mathrm{~kg} / \mathrm{m}^{2}$. La sensibilidad y especificidad indican que sólo el IMC autorreportado tuvo una mayor sensibilidad en la categoría $<25 \mathrm{~kg} / \mathrm{m}^{2}$, y una mayor especificidad en la categoría $\geq 30 \mathrm{~kg} / \mathrm{m}^{2}$. Cuando las categorías de sobrepeso y obesidad se colapsaron en una, la sensibilidad fue de $82.0 \%$ y la especificidad de $91.1 \%$ (cuadro III).

\section{Percepción de imagen corporal autorreportada}

La correlación entre las medidas del IMC y la PIC fue de 0.64 (0.67 para las mujeres y 0.59 para los hombres); ambas fueron estadísticamente significativas para quienes subestimaron el IMC $(0.73, p<0.05)$ y para quienes lo sobrestimaron $(0.68, p<0.000)$. Como se observa en

\section{Cuadro III SenSIBILIDAD Y ESPECIFICIDAD DE DATOS AUTORREPORTADOS PARA SOBREPESO Y OBESIDAD. Estudio de COHORTE del Instituto MeXicano del Seguro Social, Morelos, México, 1998}

$\begin{array}{rrr} & \text { Sensibilidad } & \text { Especificidad } \\ n & \% \text { (IC 95\%) } & \% \text { (IC 95\%) }\end{array}$

IMC autorreportado versus medido

$93470.8(70.4-71.3) \quad 78.6(78.2-78.9)$

0 besidad $\quad 60.7(59.8-61.6) \quad 98.2(98.1-98.3)$

Sobrepeso más obesidad $\quad 82.0(81.7-82.3) \quad 91.1(90.9-91.2)$

PIC versus IMC medido

Sobrepeso $\quad 93466.0(65.5-66.4) \quad 58.7(58.2-59.2)$

0 besidad $67.1(66.2-68.0) \quad 89.4(89.0-89.7)$

Sobrepeso más obesidad $\quad 87.6(87.3-87.9) \quad 48.9(48.2-49.6)$

IMC autorreportado corregido

versus medido

\begin{tabular}{|c|c|c|}
\hline Sobrepeso & 428 & $74.0(72.9-75.1)$ \\
\hline 0 besidad & & $68.7(66.3-71.0)$ \\
\hline Sobrepeso más obesidad & & $94.8(93.8-95.6)$ \\
\hline
\end{tabular}

$I M C=$ índice de masa corporal

$\mathrm{PIC}=$ percepción de imagen corporal

el cuadro II, las correlaciones entre el IMC y la PIC por ocupación fueron de 0.61 (empleados de intendencia y de multitareas) a 0.76 (pensionados); por el nivel de educación la correlación fue de 0.43 (primaria) a 0.66 (estudios profesionales y de posgrado); y por estado civil fueron de 0.34 (viudos) a 0.74 (nunca casados).

La prevalencia de obesidad (PIC $\geq 7$ ) fue de $23.9 \%$ (21.2\% para las mujeres y $28.4 \%$ para los hombres). La sensibilidad y la especificidad de la categoría de obesidad (IMC $\geq 30 \mathrm{~kg} / \mathrm{m}^{2}$ versus PIC $\geq 7$ ) fueron de $67.1 \mathrm{y}$ $89.4 \%$, respectivamente; cuando las categorías de sobrepeso y obesidad (IMC $\geq 25 \mathrm{~kg} / \mathrm{m}^{2}$ versus PIC $\geq 4$ ) se colapsaron en una, la sensibilidad fue de $87.6 \%$ y la especificidad de $48.9 \%$ (cuadro III).

\section{Modelo correctivo}

La muestra original fue de 934 sujetos. Se hizo un análisis de regresión robusta en la muestra seleccionada aleatoriamente de 506 participantes para evaluar la diferencia de los estimados de peso y talla (medido menos autorreportado) como una función del sexo, grupo de edad, nivel de educación, estado civil, ocupación, PIC y cambio en el peso durante el último año. 
En el cuadro IV se presenta el resumen de los análisis de regresión múltiple para evaluar la diferencia en los estimadores de talla y peso. Se calcularon nuevas estimaciones de talla y peso autorreportados mediante la suma de la diferencia prevista de la cual se derivó el IMC.

La correlación entre el IMC medido y el autorreportado corregido fue de 0.91 para las mujeres y de 0.93 para los hombres (cuadro II). Los valores de dicha correlación dentro de las diferentes categorías del IMC medido $\left(<25,25-30\right.$ y $\left.\geq 30 \mathrm{~kg} / \mathrm{m}^{2}\right)$ fueron de $0.74,0.66$ y 0.73 , respectivamente. La media de la diferencia entre el IMC medido y el autorreportado corregido no fue estadísticamente significativo $(p=0.94)$. De todos los participantes, $51.5 \%$ sobrestimaron el IMC autorreportado corregido (media $-1.12 \mathrm{~kg} / \mathrm{m}^{2} \pm 0.95$ ) y $48.5 \%$ lo subestimaron (media $1.29 \mathrm{~kg} / \mathrm{m}^{2} \pm 1.18$ ). No hubo diferencia significativa en la precisión de la estimación de la diferencia entre el IMC medido y el autorreportado corregido por sexo (media $0.08 \mathrm{~kg} / \mathrm{m}^{2} \pm 1.67$ para las mujeres; $0.01 \mathrm{~kg} / \mathrm{m}^{2} \pm 1.50$ para los hombres; $p=0.61$ ); entre quienes subestimaron el IMC corregido, dichas estimaciones fueron de 1.33 para las primeras, versus $1.23 \mathrm{~kg} / \mathrm{m}^{2}$ para los segundos, $p=0.84 \mathrm{y}$, para quienes lo sobrestimaron, de -1.16 versus $-1.05 \mathrm{~kg} / \mathrm{m}^{2}, p=0.42$, respectivamente.

\section{Cuadro IV \\ Coeficientes para estimar la diferencia de la talla Y DEL PESO EN FUNCIÓN DE COVARIABLES. EstUdio DE COHORTE del Instituto MeXicano del Seguro Social, Morelos, México, 1998}

\begin{tabular}{lcc} 
Variable & $\beta$ & IC 95\% \\
Talla corregida & & \\
$\quad$ Edad 60-76 años & -0.0315 & $-0.0444,-0.0186$ \\
\hline Percepción imagen corporal & 0.0006 & $-0.0004,0.0017$ \\
\hline Aumento de peso en el último año & 0.0035 & $-0.0002,0.0073$ \\
\hline Constante & -0.0148 & $-0.0205,-0.0091$
\end{tabular}

\begin{tabular}{lcr}
$\begin{array}{l}\text { Peso corregido } \\
\text { Hombres }\end{array}$ & 0.314 & $-0.242,0.869$ \\
\hline Edad 60-76 años & 1.966 & $-0.420,4.351$ \\
\hline Educación: primaria & 0.329 & $-0.259,0.918$ \\
\hline Estado civil: nunca casados & -0.537 & $-1.278,0.205$ \\
\hline Personal de salud & -0.333 & $-0.917,0.252$ \\
\hline Pensionados & -1.105 & $-2.940,0.730$ \\
\hline Percepción de imagen corporal & 0.187 & $0.043,0.331$ \\
\hline Pérdida de peso en el último año & 1.003 & $0.269,1.737$ \\
\hline Aumento de peso en el último año & -0.420 & $-0.966,0.127$ \\
\hline Constante & 0.407 & $-0.450,1.264$
\end{tabular}

La diferencia entre el IMC medido y el autorreportado corregido por nivel de educación, ocupación y estado civil no fue significativa. La distribución de la diferencia del IMC corregido tuvo una tendencia positiva a través de las diferentes categorías del IMC medido. La desviación estándar del error reportado aumentó junto con las categorías del IMC medido (1.30, 1.44 y $2.02 \mathrm{~kg} / \mathrm{m}^{2}$ para IMC $<25 ; 25-30$ y $\geq 30 \mathrm{~kg} / \mathrm{m}^{2}$, respectivamente; datos no mostrados).

El grado de clasificación errónea entre el IMC medido y el autorreportado corregido, usando diferentes categorías del IMC, se muestra en el cuadro III. La sensibilidad y la especificidad de las categorías combinadas de sobrepeso y obesidad fueron de $94.8 \%$ y $83 \%$, respectivamente.

\section{Discusión}

Los resultados muestran que las características autorreportadas, tales como peso y talla corregidos, así como la PIC -validada mediante el IMC medido-, son medidas válidas que se pueden utilizar para estimar valores categóricos y continuos del IMC en población adulta mexicana, en vista de la sensibilidad obtenida para la categoría sobrepeso más obesidad. El presente análisis también permitió identificar los factores sociodemográficos más relevantes que pueden usarse para corregir la diferencia potencial en el peso y la talla autorreportados. Su utilización mejorará considerablemente la precisión de estos datos que sugieren que su procedimiento se puede usar en otros estudios en diferentes poblaciones.

La correlación observada para la talla medida y autorreportada ( $r=0.94$ ) es consistente con las establecidas en estudios previos ( $r=0.94$ a 0.97$) .6,13,33$ Stewart y colaboradores, ${ }^{14}$ en una muestra de 1598 individuos de Nueva Zelanda, encontraron una diferencia absoluta en talla de no más de $3.5 \mathrm{~cm}$, mientras que en el presente estudio la media de la diferencia fue de $1.3 \mathrm{~cm}$ o menos. A pesar de que el error de esta estimación es más pequeño que el obtenido por otros investigadores, el modelo de ajuste puede reducir la media de la diferencia a $1.1 \mathrm{~cm}$. De manera consistente con otros estudios, se encontró que las personas con sobrepeso tienden a sobrestimar su talla. ${ }^{6}$ En la población de estudio, la diferencia en la talla percibida se debió principalmente a la edad, al aumento de peso y a la PIC.

La correlación observada entre el peso medido y el autorreportado $(r=0.96)$ en esta población es consistente con informes previos relacionados con la validez del peso estimado de esta manera en países industrializados ( $r=0.91$ a 0.98$) .6,13,33,34$ Lissner y colaboradores encontraron una correlación de $r=0.94$ para peso auto- 
rreportado y medido entre adultos obesos de Suecia, similar al valor de correlación de 0.95 en esa categoría de la población del presente estudio.

Stewart y colaboradores ${ }^{14}$ encontraron que la diferencia absoluta en peso no excedió de $2.4 \mathrm{~kg}$, mientras que en este estudio no excedió los $3.17 \mathrm{~kg}$. Este error en la estimación estuvo influido por algunas variables que se consideraron en el modelo para corregir el peso autorreportado.

Diversos investigadores señalan que los hombres maduros tienden a subestimar su peso,6,12,13,35,36 nuestros resultados son consistentes con lo anterior y mostraron que los errores en el peso así valorado estuvieron directamente relacionados con el sobrepeso de los individuos, un hallazgo también señalado por Rowland, quien estudió una muestra de 11284 adultos de edades de entre 20 a 76 años, en la National Health and Nutrition Examination Survey II (NHANES II), de Estados Unidos de América. ${ }^{9}$

En el presente estudio, la correlación observada entre el IMC medido y el autorreportado fue de $r=0.90$, un valor entre los límites de los informados por Stewart y colaboradores ${ }^{14}$ y por Reed y Price, ${ }^{33}$ de $r=0.80$ y 0.93 , respectivamente. El valor de la correlación aumentó a 0.91 cuando se evaluó la correlación entre el IMC medido y el autorreportado corregido.

Algunos autores han tratado de validar la autopercepción de la imagen corporal como un indicador del grado de sobrepeso. ${ }^{37-42}$ Madrigal-Fritsch y colaboradores, ${ }^{42}$ compararon la PIC con el IMC derivado del peso y la talla autorreportados en población española. Ellos encontraron que, de acuerdo con la PIC, las mujeres estimaron la obesidad en mayor proporción que los hombres. En nuestra población los resultados fueron opuestos. Es importante señalar que nuestros puntos de corte para clasificar sobrepeso, usando siluetas, son diferentes de aquellos usados por Madrigal-Fritsch y colaboradores; ésta pudiera ser la razón de tales diferencias. También es posible asignar un valor medio al IMC e intervalos de confianza a cada una de las siluetas. Sin embargo, se observó que en las siluetas 1 y 9, los valores medios del IMC no están de acuerdo con los esperados, posiblemente debido a la baja proporción de individuos clasificados dentro de estas dos categorías.

Este estudio muestra que los altos valores de sensibilidad y especificidad se obtienen cuando se colapsan las categorías de sobrepeso y obesidad en una, valores que se pueden usar para ajustar razones de momios y riesgos relativos; por tanto, remover la diferencia causó la clasificación errónea debido al uso de los valores autorreportados más que de los medidos. ${ }^{43,44}$

Deben considerarse algunas limitaciones al interpretar estos resultados. Las medidas de peso se rea- lizaron entre 4 y 8 meses después del autorreporte; sin embargo, para controlar cambios potenciales en peso en el tiempo, se preguntó a los sujetos del estudio si su peso se había modificado durante el último año. Ante respuesta afirmativa se solicitó más información acerca de peso ganado o perdido, la cual fue utilizada como una covariable en los modelos para corregir el peso y talla autorreportados.

Debido a las características de la población en este estudio -los trabajadores de la salud tienen más probabilidad de tener medidas frecuentes de talla y peso-, estos hallazgos deben ser validados en otras poblaciones, por ejemplo entre analfabetas o entre personas con acceso limitado a los servicios de atención a la salud en población mexicana.

\section{Reconocimientos}

Apreciamos la colaboración de Salvador Zamora Muñoz, Alma E. López Caudana, Patricia Espinosa, Laura Martinell y Sergio Juárez, en las diferentes etapas de este estudio. Estamos especialmente agradecidos con Magda Luz Atrián Salazar por su apoyo en la preparación del manuscrito. También estamos en deuda con todos los participantes del estudio y el personal involucrados en la obtención de los datos y en la dirección del "Estudio de cohorte del IMSS".

\section{Referencias}

1. Popkin MB, Doak MC. Nutr Rev 1998;56(4):106-114. 2. Pi-Sunyer FX. Health implications of obesity. Am J C lin N utr 1991;53:1595S-1603S.

3. Pi-Sunyer. Medical hazards of obesity.Ann Intern Med 1993;119:655-660. 4. $\mathrm{N}$ ational Institutes of $\mathrm{H}$ ealth. $\mathrm{N}$ ational $\mathrm{H}$ eart, Lung, and Blood Institute. Clinical guidelines on the identification, evaluation, and treatment of overweight and obesity in adults -The evidence Report. 0 bes Res 1998;6 Suppl 2:51S, 795.

5. González-Villalpando C, Stern MP. La obesidad como factor de riesgo cardiovascular en México. Estudio en población abierta. Rev Invest Clin 1993;45:13-21.

6. Stewart AL.The reliability and validity of self-reported weight and height. J Chronic Dis 1982;35:295-309.

7. Sorensen TIA, Stunkard AJ, Teasdale TW, Higgins MW. The accuracy of report of weight: Children recall of their parent's weight 15 years earlier. Int I 0 bes 1983; 7:115-122.

8. Lissner L, Sjstrom L, Bengetsson C, Bouchard C, Larsson B. The natural history of obesity in an obese population and associations with metabolic aberrations. Int J 0 bes Relat Metab Disord 1994;18(6):441-447.

9. Rowland ML. Reporting bias in height and weight data. Stat Bull $1989 ; 70: 2-8$

10. Rowland ML. Self-reported weight and height. A m J C lin N utr 1990;52:1125-1133.

11. Schlinchting $P$, Hoilund-C arlsen PF, Q uaade F, et al. C omparison of self-reported height and weight with controlled height and weight in women and men. Int J 0 bes 1981;5:67-76. 
12. Pirie $P$, Jacobs $D$, Jeffery $R$, et al. D istortion in self-reported height and weight data. I Am Diet Assoc 1981;78:601-606.

13. Palta M, Prineas RJ, Berman R, H annan P. Comparison of selfreported and measured height and weight.Am J Epidemiol 1982;115:223-230

14. Stewart AW, Jackson RT, Ford MA, Beaglehole R. Underestimation of relative weight by use of self-reported height and weight.Am J Epidemiol 1987;125:122-126.

15. Davis H, Gergen PJ.The weight and height of Mexican-American adolescents:The accuracy of self-reports. Am J Pub Health 1994:84(3):459-462.

16. Lee SK.Validity of self-reported weight and height: Comparison between immigrant and non-immigrant Mexican Americans in N HAN ES III. J Immigr Health 2005;7(2):127-31.

17. Avila-Funes JA, Gutierrez-Robledo LM, Ponce De Leon Rosales S. Validity of height and weight self-report in Mexican adults: Results from the national health and aging study. J N utr Health Aging 2004;8(5):355-361.

18. Santillan AA, C amargo CA. Body mass index and asthma among Mexican adults:The effect of using self-reported vs. measured weight and height. Int I 0 bes Relat Metab D isord 2003;27(11):1430-1433. 19. Hauck FR, W hite L, C ao G, W oolf N, Strauss K. Inaccuracy of selfreported weights and heights among American Indian adolescents. Arch Environ Health 2002:57(5):450-460.

20. D avis H, Gergen PJ. Self-described weight status of MexicanAmerican adolescents. J Adolesc Health 1994;15(5):407-409.

21. D avis H, Gergen P. The weights and heights of Mexican-American adolescents:The accuracy of self-reports. Am J Clin N utr 1990;52(6):1125-1133.

22. Q uetelet LA. Physique sociale. Bruselas: Real A cademia de Bélgica, 1997. 23. Bray GA, Bouchard C, James W P. D efinition and proposed current classification of obesity. En: Handbook of obesity. N ew York: Marcel Dekker Inc., 1997:31-32.

24. 0 rganización Mundial de la Salud. 0 besity: Preventing and managing the global epidemic. Report of aW HO consultation on obesity. G inebra: O MS, 1997:3-5. Division of N oncommunicable Diseases, Programme of Nutrition, Family and Reproductive Health.W HO /N UT/N CD/98.1. 25.W orld Health $O$ rganization. Physical status:The use and interpretation of anthropometry. Report of W HO expert committee. [N 0.845]. G inebra:W HO , 1995:368-369.

26. Rosner B. Fundamentals of biostatistics. 4a edición. N ueva York: W iley \& Sons, 1995.

27. Stevens J, Keil JE,W aid LR, G azes PC.Accuracy of current, 4-year, and 28-year self-reported body weight in an elderly population. Am J Epidemiol 1990;132(6):1156-1163.
28. Hoaglin DC, Mosteller F, Tukey JW. Understanding robust and exploratory data analysis. N ueva York: John W iley and Sons Inc., 1983. 29.W eisberg S.A pplied linear regression. 2a. edición. N ueva York:W iley $\&$ Sons, 1985.

30. Everitt BS. The analysis of contingency tables. 2a. edición. Londres: Chapman \& Hall, 1991:37-59.

31. Coughlin SS, Trock B, Criqui MH, Pickle LW, Browner D, Teefft M. The logistic modeling of sensitivity, specificity, and predictive value of a diagnostic test. J C lin Epidemiol 1992;45(1):1-7.

32. Choi BCK. Sensitivity and specificity of a single diagnostic test in the presence of work-up bias. J Clin Epidemiol 1992;45(6):581-586. 33. Reed DR, Price RA. Estimates of the weight and height of family members:Accuracy of informant reports. Int J 0 bes 1998;22(9):827-835 34. Sturkard AJ,A Ibaum JM.The accuracy of self-reported weight. Am J Clin N utr 1981;34:1593-1599.

35. Charney EG, Chanblee H, MCBride M. Childhood antecedents of adult obesity. N Engl J Med 1976;295:6-9.

36.W ing RR, Epstein LH, 0 ssip DJ. Reliability and validity of self-report and observed estimates of relative weights. Addict Behav 1979;4:113-140. 37. Davis C, D urnin JV, D ionne M, G urevich M. The influence of body fat content and bone diameter measurements on body dissatisfaction in adult women. Int J Eat Disord 1994;15:257-263.

38. G ittelso hn J, Harris SB, Lyman TH, Hanley A, Barnie A, Zinman B. Body image concepts differ by age and sex in an 0 jibway-Cree community in Canada. J N utr 1996;126:2990-3000.

39. Grilo CM, W ilfley DE, Brownell KD, Rodin J.Teasing, body image, and self-esteem in a clinical sample of obese women. Addict Behav 1994:19:443-450.

40. Sisson BA, Franco SM, C arlin W M, Mitchell CK. Body fat analysis and perception of body image. C lin Pediatr (Phila) 1997;36:415-418.

41. Cachelin FM, Striegel RH, Elder KA. Realistic weight perception and body size assessment in a racially diverse community sample of dieters. 0 bes Res 1998;6:52-68.

42. Madrigal-Fritsch H, Irala-Estévez J, Martínez-G onzález MA, Kearney J, Gibney M, Martínez-Hernández JA. Percepción de la imagen corporal como aproximación cualitativa al estado de nutrición. Salud Publica Mex 1999;41(6):479-486

43. Copeland KT, Checkoway H, MCMichael AJ, Holbrook RH. Bias due to misclassification in the estimation of relative risk. Am J Epidemiol 1977; 105:488-495

44. G reenland S.The effect of misclassification in the presence of covariates. Am J Epidemiol 1980;112:564-569. 